Network Working Group

Request for Comments: 2580

STD : 58

Obsoletes: 1904

Category: Standards Track
Editors of this version:

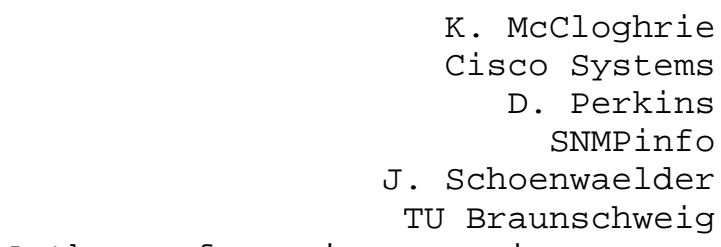

Authors of previous version:

J. Case

SNMP Research

K. McCloghrie

Cisco Systems

M. Rose

First Virtual Holdings

S. Waldbusser

International Network Services

April 1999

\title{
Conformance Statements for SMIv2
}

Status of this Memo

This document specifies an Internet standards track protocol for the Internet community, and requests discussion and suggestions for improvements. Please refer to the current edition of the "Internet Official Protocol standards" (STD 1) for the standardization state and status of this protocol. Distribution of this memo is unlimited.

Copyright Notice

Copyright (C) The Internet Society (1999). All Rights Reserved.

Table of Contents

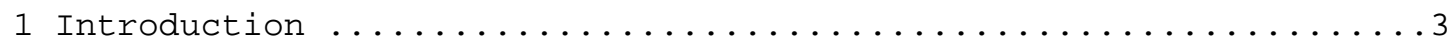

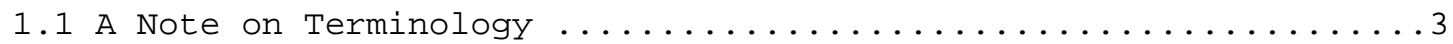

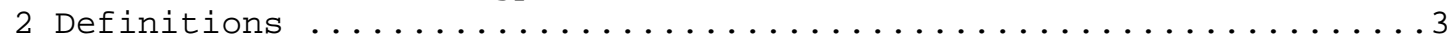

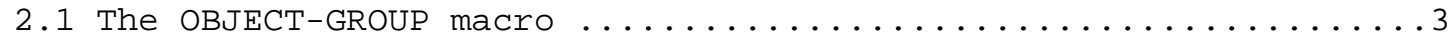

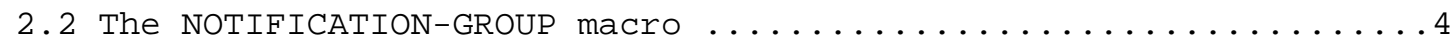

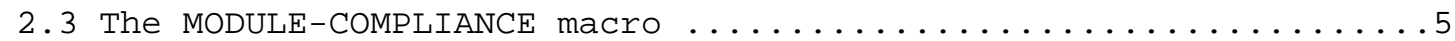

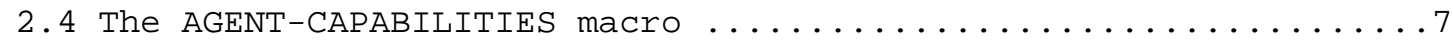

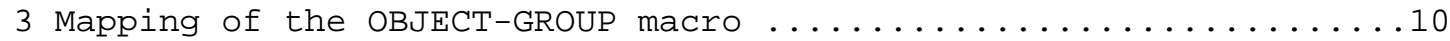

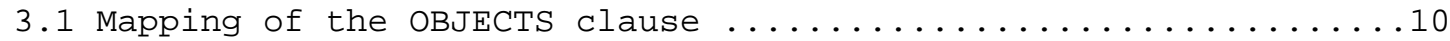

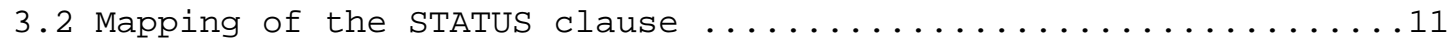

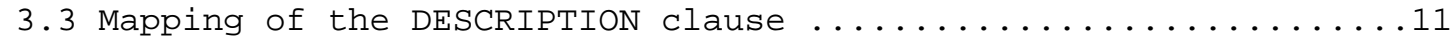

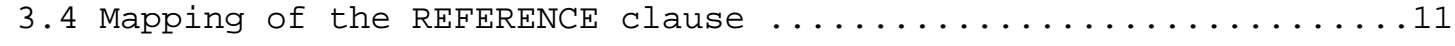




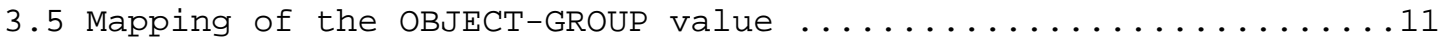

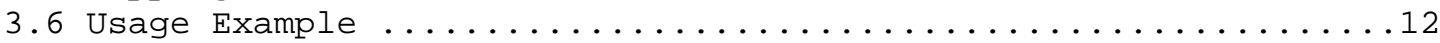

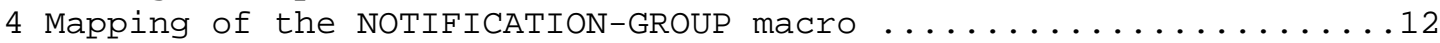

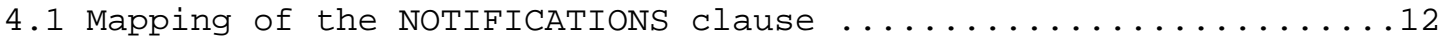

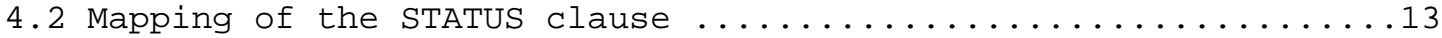

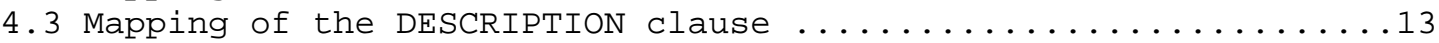

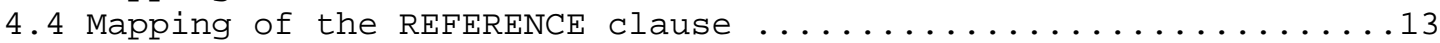

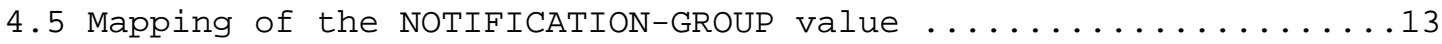

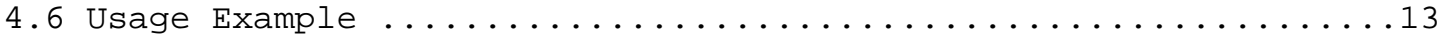

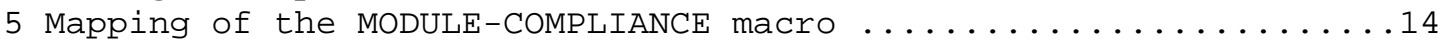

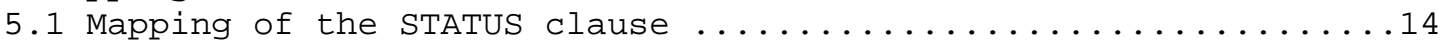

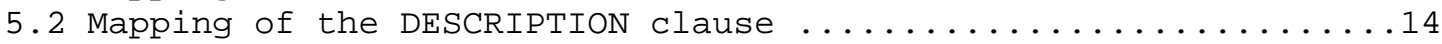

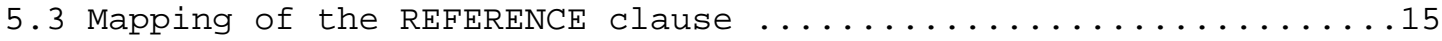

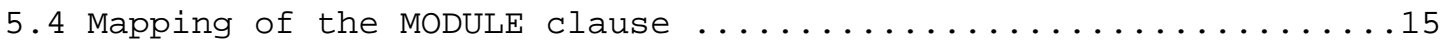

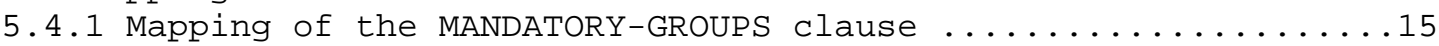

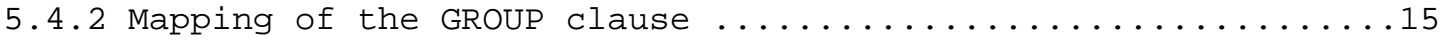

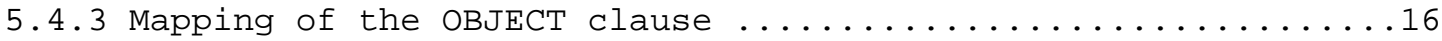

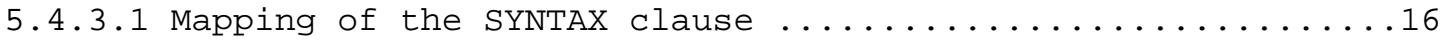

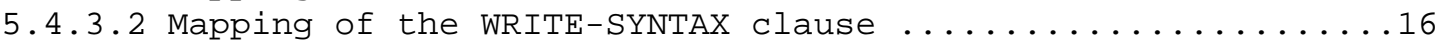

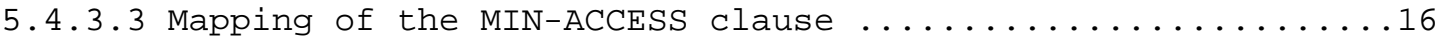

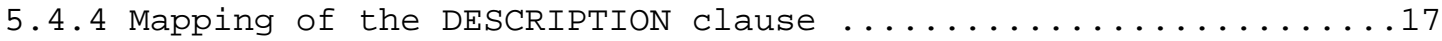

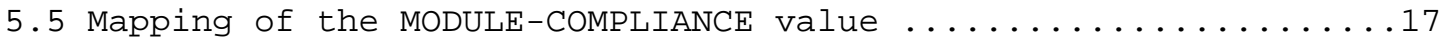

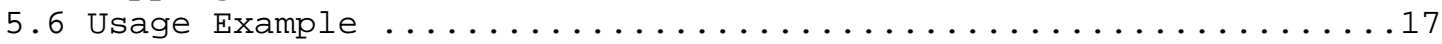

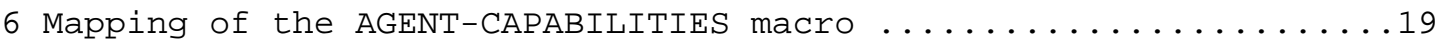

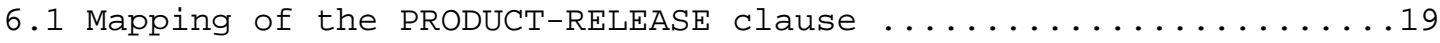

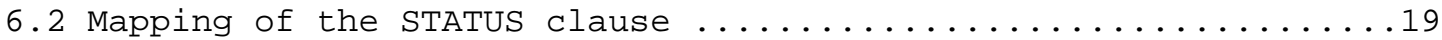

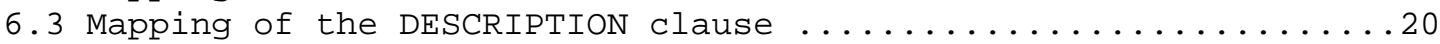

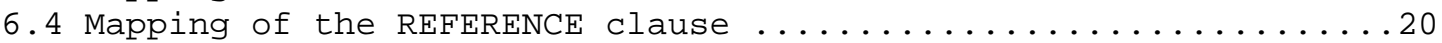

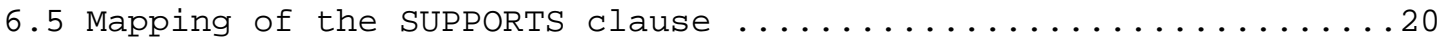

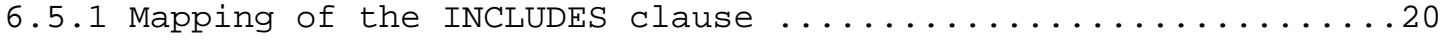

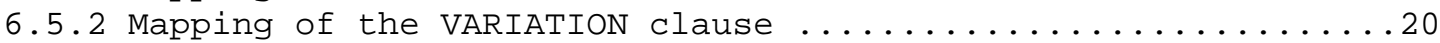

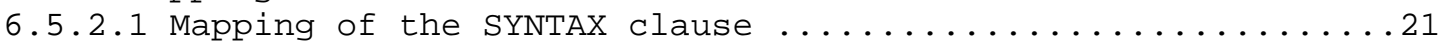

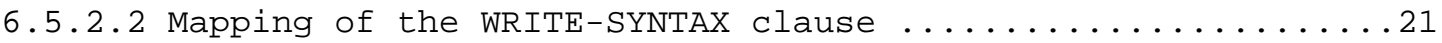

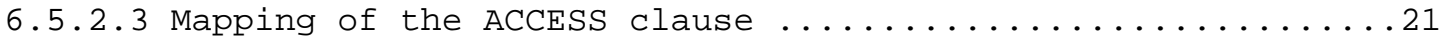

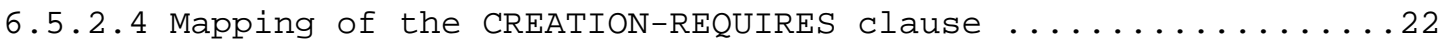

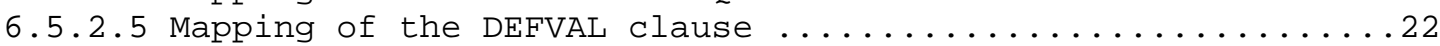

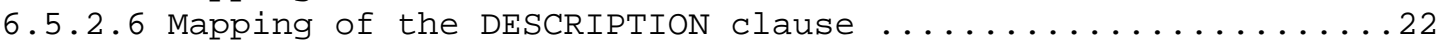

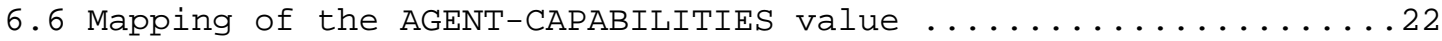

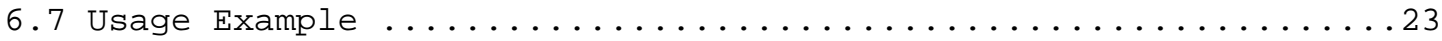

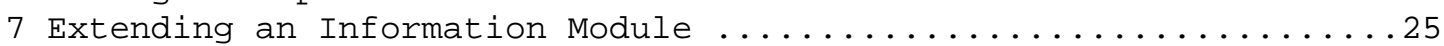

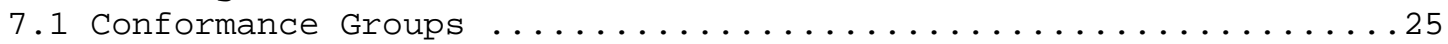

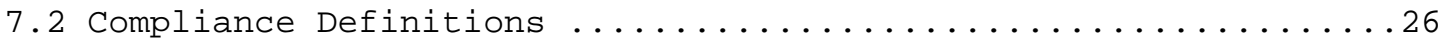

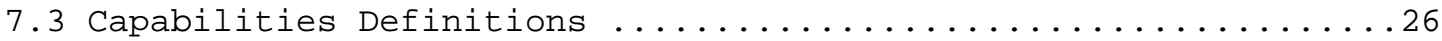

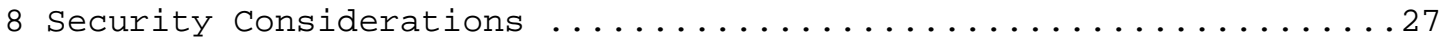

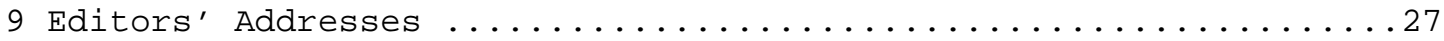

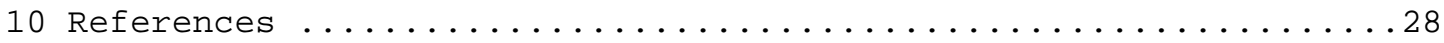

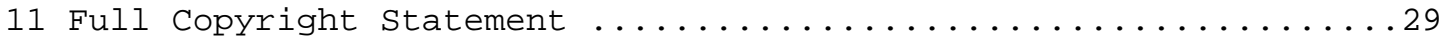




\section{Introduction}

Management information is viewed as a collection of managed objects, residing in a virtual information store, termed the Management Information Base (MIB). Collections of related objects are defined in MIB modules. These modules are written using an adapted subset of OSI'S Abstract Syntax Notation One, ASN.1 (1988) [1], termed the Structure of Management Information (SMI) [2].

It may be useful to define the acceptable lower-bounds of implementation, along with the actual level of implementation achieved. It is the purpose of this document to define the notation used for these purposes.

1.1. A Note on Terminology

For the purpose of exposition, the original Structure of Management Information, as described in RFCs 1156 (STD 16), 1212 (STD 16), and RFC 1215, is termed the SMI version 1 (SMIV1). The current version of the structure of Management Information is termed SMI version 2 (SMIV2).

2. Definitions

SNMPV2-CONF DEFINITIONS : := BEGIN

IMPORTS ObjectName, NotificationName, objectsyntax

FROM SNMPV2-SMI;

-- definitions for conformance groups

OBJECT-GROUP MACRO : :=

BEGIN

TYPE NOTATION : :=

Objectspart

"STATUS" Status

"DESCRIPTION" Text

Referpart

VALUE NOTATION : :=

value (VALUE OBJECT IDENTIFIER)

Objectspart : :=

Objects : :=

"OBJECTS" "\{" Objects " $\}$ "

Object : : =

Object

| Objects "," Object

McCloghrie, et al. 


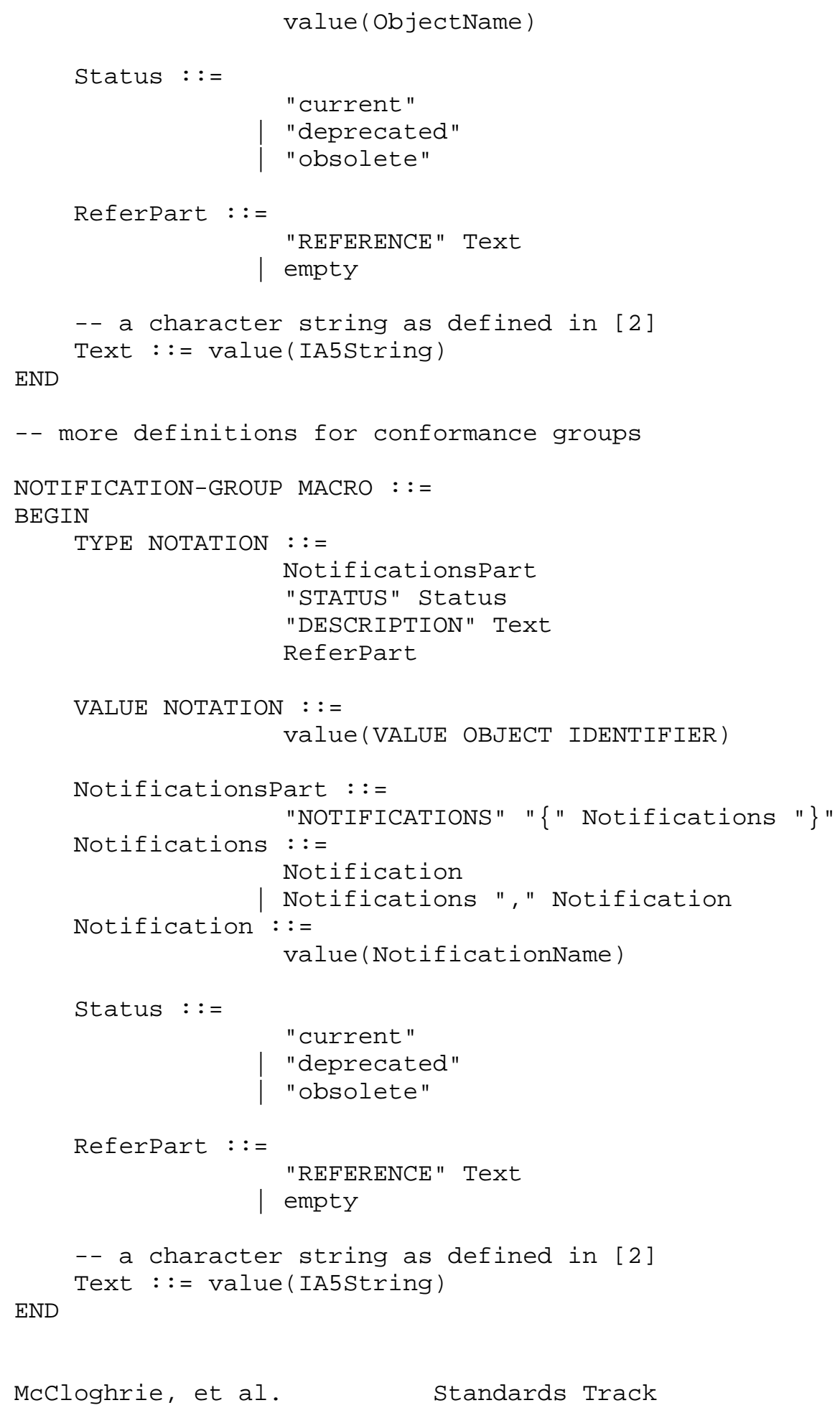

McCloghrie, et al. Standards Track 


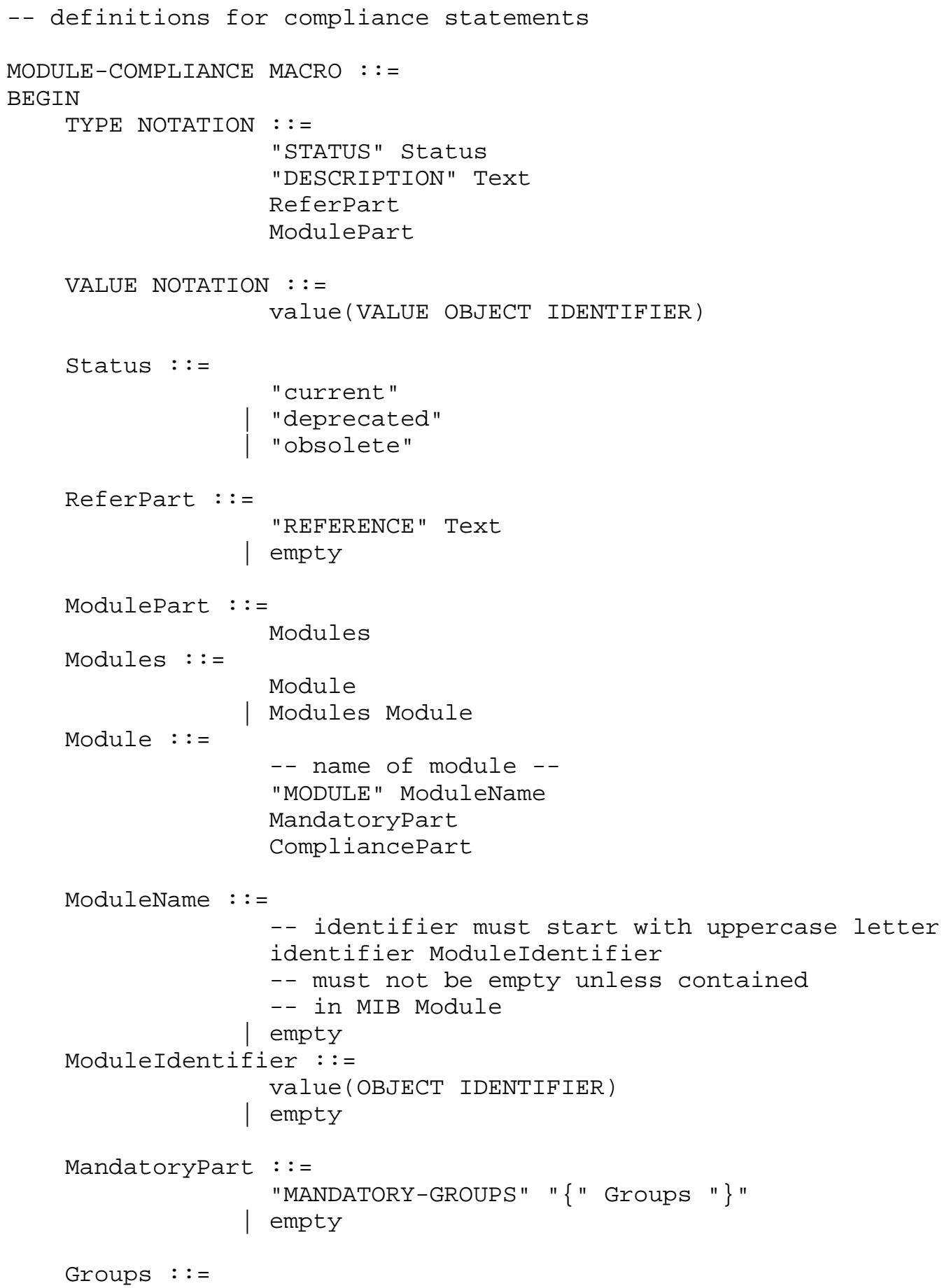




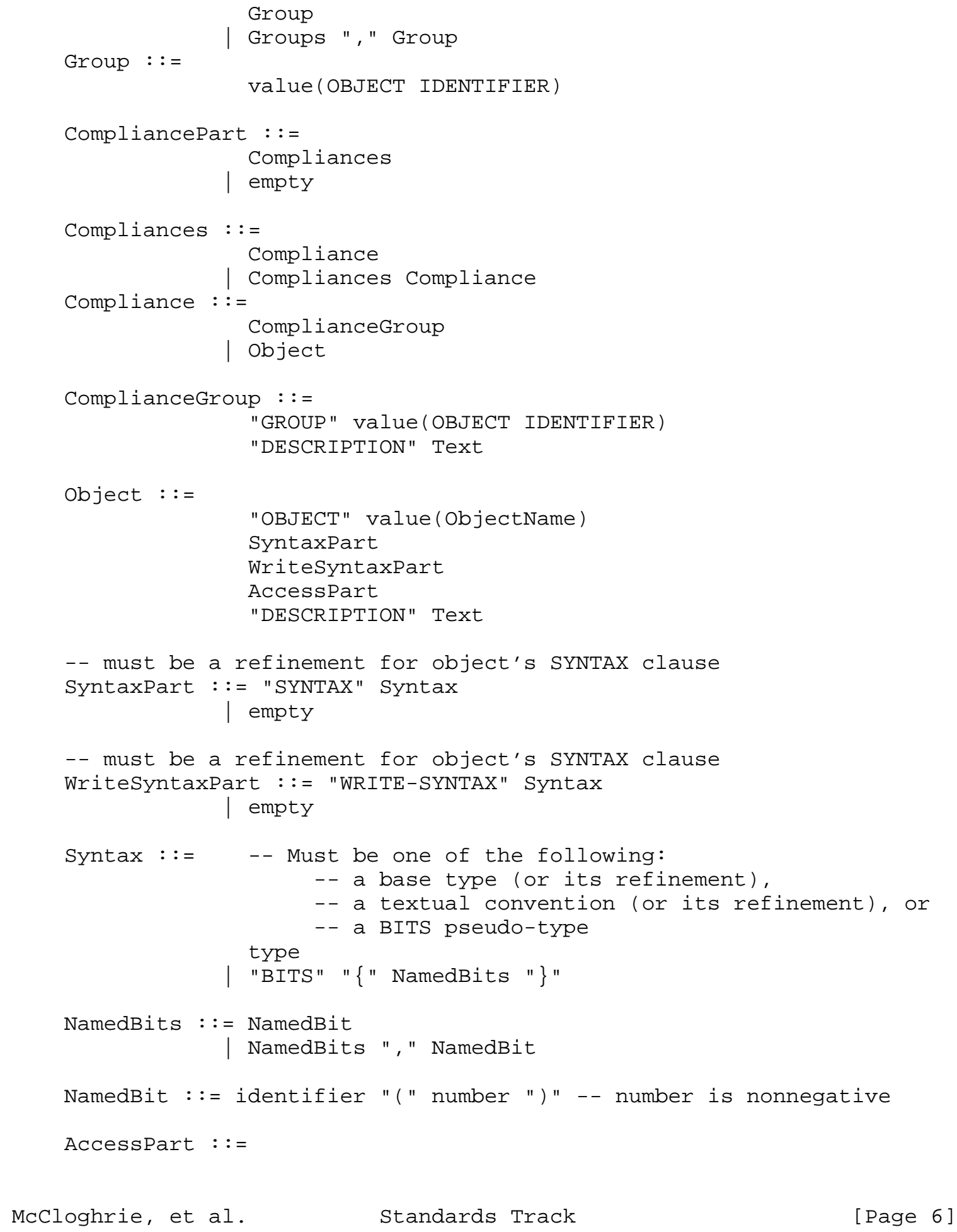




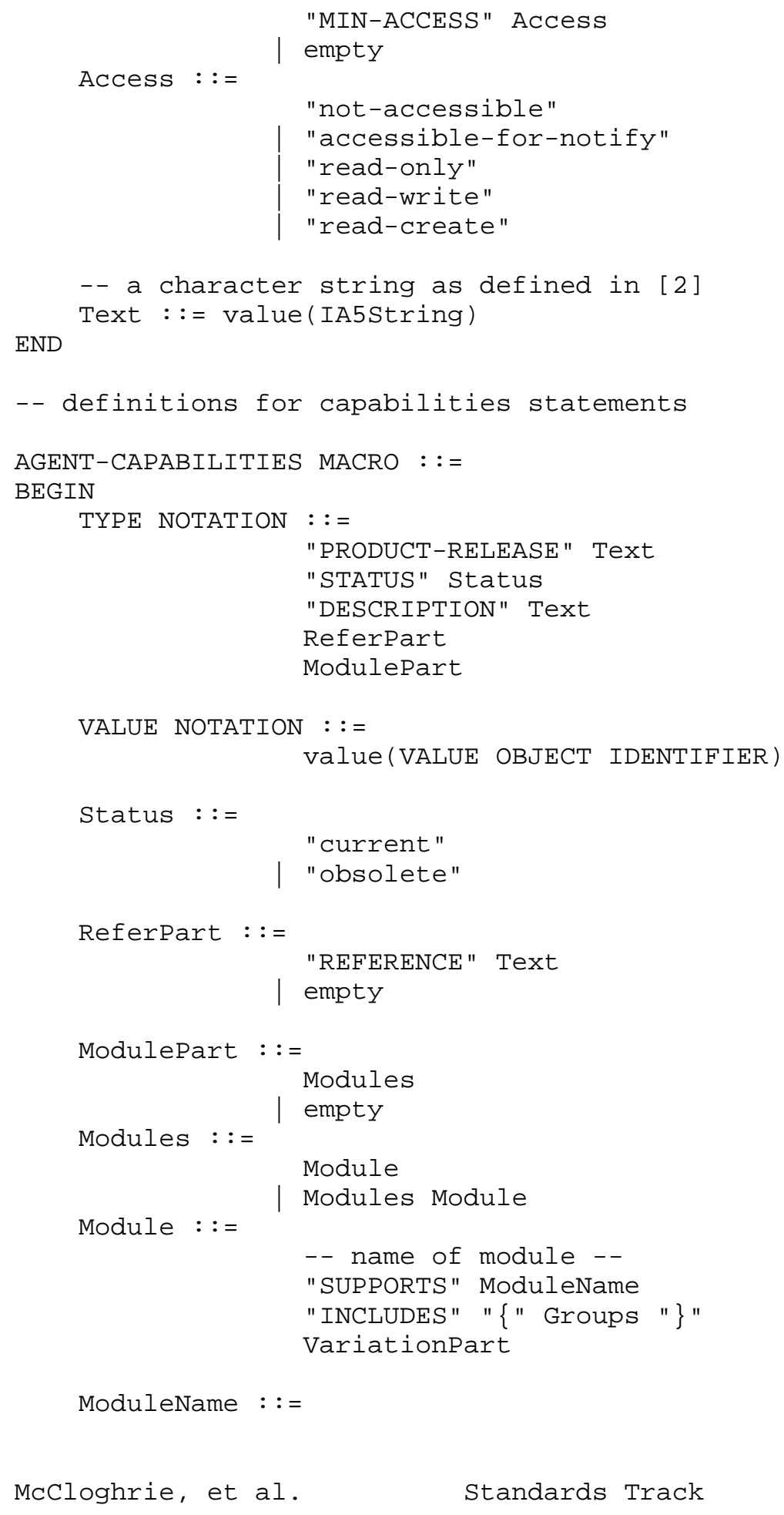




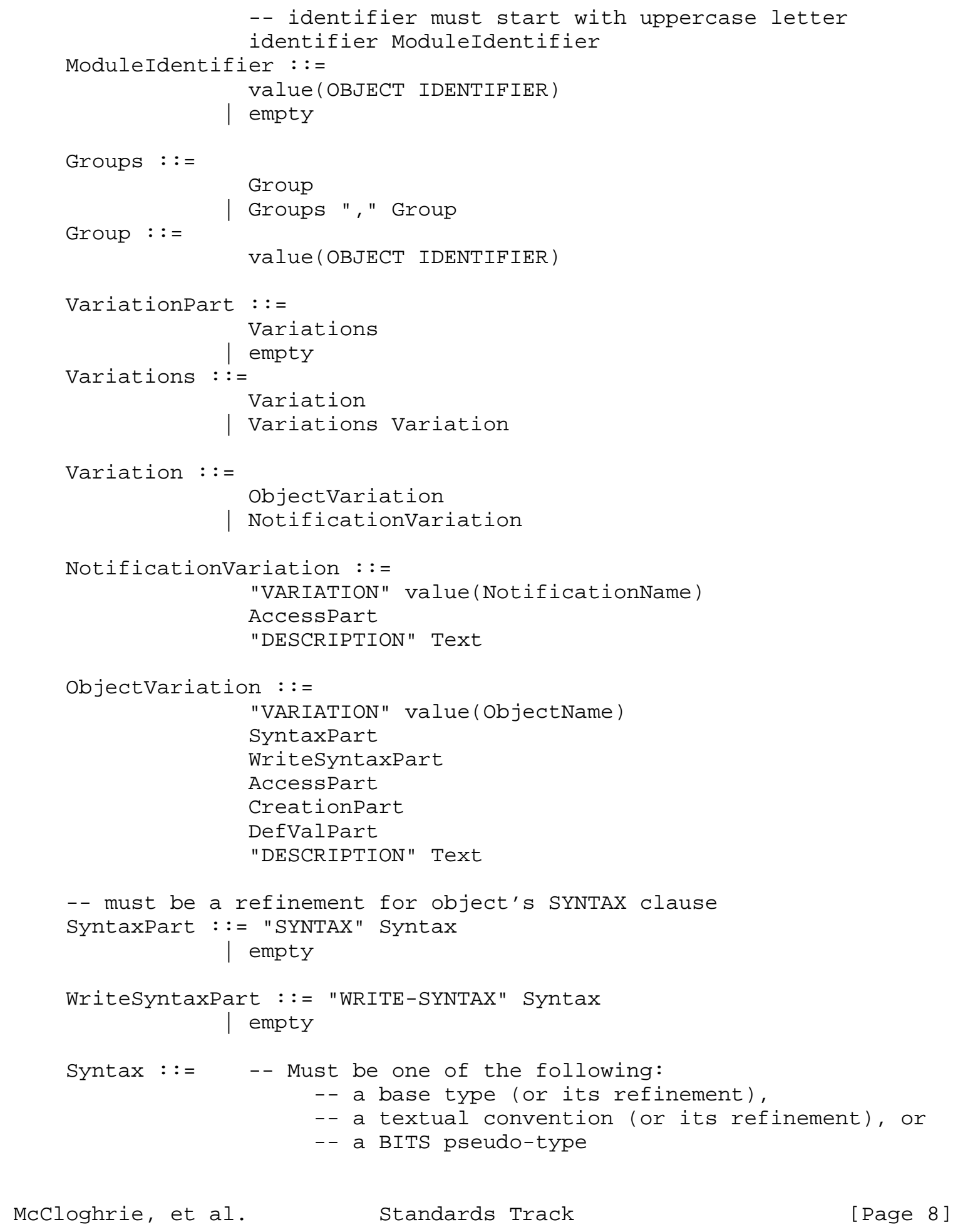




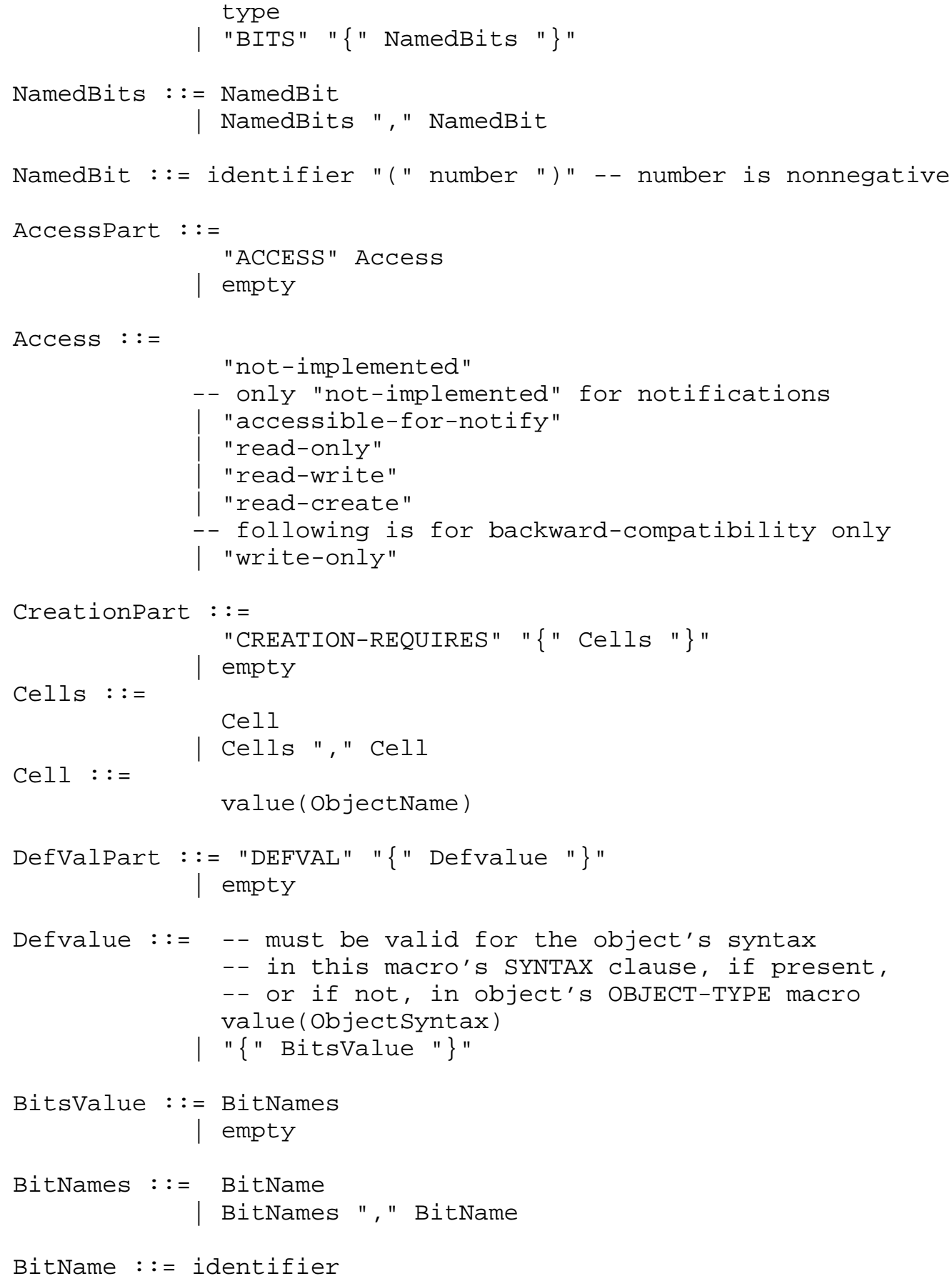


-- a character string as defined in [2]

END

Text : := value (IA5String)

END

3. Mapping of the OBJECT-GROUP macro

For conformance purposes, it is useful to define a collection of related managed objects. The OBJECT-GROUP macro is used to define each such collection of related objects. It should be noted that the expansion of the OBJECT-GROUP macro is something which conceptually happens during implementation and not during run-time.

To "implement" an object, an agent must return a reasonably accurate value for management protocol retrieval operations; similarly, if the object is writable, then in response to a management protocol set operation, an agent must accordingly be able to reasonably influence the underlying managed entity. If an agent can not implement an object, the management protocol provides for it to return an exception or error, e.g, noSuchobject [4]. Under no circumstances shall an agent return a value for objects which it does not implement -- it must always return the appropriate exception or error, as described in the protocol specification [4].

Note that the OBJECT-GROUP macro itself provides no conformance information. Rather, conformance information is specified through the inclusion of defined groups in a MODULE-COMPLIANCE macro.

\subsection{Mapping of the OBJECTS clause}

The OBJECTS clause, which must be present, is used to specify each object contained in the conformance group. Each of the specified objects must be defined in the same information module as the OBJECT-GROUP macro appears, and must have a MAX-ACCESS clause value of "accessible-for-notify", "read-only", "read-write", or "readcreate".

It is required that every object defined in an information module with a MAX-ACCESS clause other than "not-accessible" be contained in at least one object group. This avoids the common error of adding a new object to an information module and forgetting to add the new object to a group. 


\subsection{Mapping of the STATUS clause}

The STATUS clause, which must be present, indicates whether this definition is current or historic.

The value "current" means that the definition is current and valid. The value "obsolete" means the definition is obsolete and the group should no longer be used for defining conformance. While the value "deprecated" also indicates an obsolete definition, it permits new/continued use of conformance definitions using this group.

\subsection{Mapping of the DESCRIPTION clause}

The DESCRIPTION clause, which must be present, contains a textual definition of that group, along with a description of any relations to other groups. Note that generic compliance requirements should not be stated in this clause. However, implementation relationships between this group and other groups may be defined in this clause.

3.4. Mapping of the REFERENCE clause

The REFERENCE clause, which need not be present, contains a textual cross-reference to some other document, either another information module which defines a related assignment, or some other document which provides additional information relevant to this definition.

\subsection{Mapping of the OBJECT-GROUP value}

The value of an invocation of the OBJECT-GROUP macro is the name of the group, which is an OBJECT IDENTIFIER, an administratively assigned name. 


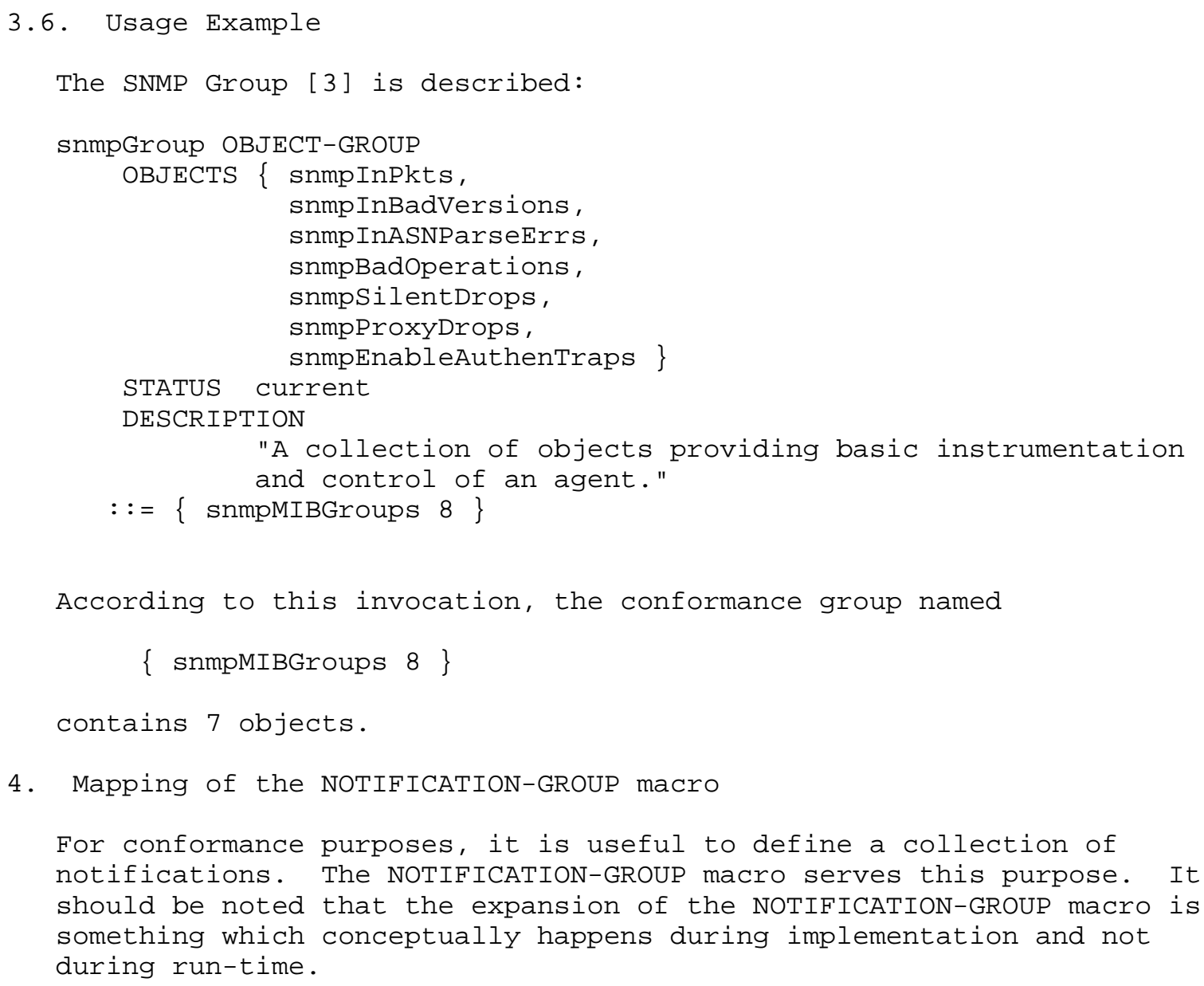




\subsection{Mapping of the STATUS clause}

The STATUS clause, which must be present, indicates whether this definition is current or historic.

The value "current" means that the definition is current and valid. The value "obsolete" means the definition is obsolete and this group should no longer be used for defining conformance. While the value "deprecated" also indicates an obsolete definition, it permits new/continued use of conformance definitions using this group.

\subsection{Mapping of the DESCRIPTION clause}

The DESCRIPTION clause, which must be present, contains a textual definition of the group, along with a description of any relations to other groups. Note that generic compliance requirements should not be stated in this clause. However, implementation relationships between this group and other groups may be defined in this clause.

\subsection{Mapping of the REFERENCE clause}

The REFERENCE clause, which need not be present, contains a textual cross-reference to some other document, either another information module which defines a related assignment, or some other document which provides additional information relevant to this definition.

\subsection{Mapping of the NOTIFICATION-GROUP value}

The value of an invocation of the NOTIFICATION-GROUP macro is the name of the group, which is an OBJECT IDENTIFIER, an administratively assigned name.

\subsection{Usage Example}

The SNMP Basic Notifications Group [3] is described: 
snmpBasicNotificationsGroup NOTIFICATION-GROUP

NOTIFICATIONS \{ coldStart, authenticationfailure \}

STATUS current

DESCRIPTION

"The two notifications which an agent is required to implement."

$::=\{$ snmpMIBGroups 7$\}$

According to this invocation, the conformance group named

$\{$ snmpMIBGroups 7$\}$

contains 2 notifications.

5. Mapping of the MODULE-COMPLIANCE macro

The MODULE-COMPLIANCE macro is used to convey a minimum set of requirements with respect to implementation of one or more MIB modules. It should be noted that the expansion of the MODULECOMPLIANCE macro is something which conceptually happens during implementation and not during run-time.

A requirement on all "standard" MIB modules is that a corresponding MODULE-COMPLIANCE specification is also defined, either in the same information module or in a companion information module.

5.1. Mapping of the STATUS clause

The STATUS clause, which must be present, indicates whether this definition is current or historic.

The value "current" means that the definition is current and valid. The value "obsolete" means the definition is obsolete, and this MODULE-COMPLIANCE specification no longer specifies a valid definition of conformance. While the value "deprecated" also indicates an obsolete definition, it permits new/continued use of the MODULE-COMPLIANCE specification.

\subsection{Mapping of the DESCRIPTION clause}

The DESCRIPTION clause, which must be present, contains a textual definition of this compliance statement and should embody any information which would otherwise be communicated in any ASN.1 commentary annotations associated with the statement. 


\subsection{Mapping of the REFERENCE clause}

The REFERENCE clause, which need not be present, contains a textual cross-reference to some other document, either another information module which defines a related assignment, or some other document which provides additional information relevant to this definition.

\subsection{Mapping of the MODULE clause}

The MODULE clause, which must be present, is repeatedly used to name each MIB module for which compliance requirements are being specified. Each MIB module is named by its module name, and optionally, by its associated OBJECT IDENTIFIER as well. The module name can be omitted when the MODULE-COMPLIANCE invocation occurs inside a MIB module, to refer to the encompassing MIB module.

\subsubsection{Mapping of the MANDATORY-GROUPS clause}

The MANDATORY-GROUPS clause, which need not be present, names the one or more object or notification groups within the correspondent MIB module which are unconditionally mandatory for implementation. If an agent claims compliance to the MIB module, then it must implement each and every object and notification within each conformance group listed. That is, if an agent returns a nosuchobject exception in response to a management protocol get operation [4] for any object within any mandatory conformance group for every possible MIB view, or if the agent cannot generate each notification listed in any conformance group under the appropriate circumstances, then that agent is not a conformant implementation of the MIB module.

\subsubsection{Mapping of the GROUP clause}

The GROUP clause, which need not be present, is repeatedly used to name each object and notification group which is conditionally mandatory for compliance to the MIB module. The GROUP clause can also be used to name unconditionally optional groups. A group named in a GROUP clause must be absent from the correspondent MANDATORYGROUPS clause.

Conditionally mandatory groups include those which are mandatory only if a particular protocol is implemented, or only if another group is implemented. A GROUP clause's DESCRIPTION specifies the conditions under which the group is conditionally mandatory.

A group which is named in neither a MANDATORY-GROUPS clause nor a GROUP clause, is unconditionally optional for compliance to the MIB module. 


\subsubsection{Mapping of the OBJECT clause}

The OBJECT clause, which need not be present, is repeatedly used to specify each MIB object for which compliance has a refined requirement with respect to the MIB module definition. The MIB object must be present in one of the conformance groups named in the correspondent MANDATORY-GROUPS clause or GROUP clauses.

By definition, each object specified in an OBJECT clause follows a MODULE clause which names the information module in which that object is defined. Therefore, the use of an IMPORTS statement, to specify from where such objects are imported, is redundant and is not required in an information module.

5.4.3.1. Mapping of the SYNTAX clause

The SYNTAX clause, which need not be present, is used to provide a refined SYNTAX for the object named in the correspondent OBJECT clause. Note that if this clause and a WRITE-SYNTAX clause are both present, then this clause only applies when instances of the object named in the correspondent OBJECT clause are read.

Consult section 9 of [2] for more information on refined syntax.

5.4.3.2. Mapping of the WRITE-SYNTAX clause

The WRITE-SYNTAX clause, which need not be present, is used to provide a refined SYNTAX for the object named in the correspondent OBJECT clause when instances of that object are written.

Consult Section 9 of [2] for more information on refined syntax.

5.4.3.3. Mapping of the MIN-ACCESS clause

The MIN-ACCESS clause, which need not be present, is used to define the minimal level of access for the object named in the correspondent OBJECT clause. If this clause is absent, the minimal level of access is the same as the maximal level specified in the correspondent invocation of the OBJECT-TYPE macro. If present, this clause must not specify a greater level of access than is specified in the correspondent invocation of the OBJECT-TYPE macro.

The level of access for certain types of objects is fixed according to their syntax definition. These types include: conceptual tables and rows, auxiliary objects, and objects with the syntax of Counter32, Counter64 (and possibly, certain types of textual conventions). A MIN-ACCESS clause should not be present for such 
objects.

An implementation is compliant if the level of access it provides is greater or equal to the minimal level in the MODULE-COMPLIANCE macro and less or equal to the maximal level in the OBJECT-TYPE macro.

5.4.4. Mapping of the DESCRIPTION clause

The DESCRIPTION clause must be present for each use of the GROUP or OBJECT clause. For an OBJECT clause, it contains a textual description of the refined compliance requirement. For a GROUP clause, it contains a textual description of the conditions under which the group is conditionally mandatory or unconditionally optional.

\subsection{Mapping of the MODULE-COMPLIANCE value}

The value of an invocation of the MODULE-COMPLIANCE macro is an OBJECT IDENTIFIER. As such, this value may be authoritatively used when referring to the compliance statement embodied by that invocation of the macro.

\subsection{Usage Example}

The compliance statement contained in the (hypothetical) XYZv2-MIB might be:

xyzMIBCompliance MODULE-COMPLIANCE

STATUS current

DESCRIPTION

"The compliance statement for XYZv2 entities which implement the XYZv2 MIB."

MODULE -- compliance to the containing MIB module MANDATORY-GROUPS \{ xyzSystemGroup,

xyzStatsGroup, xyzTrapGroup, xyzSetGroup, xyzBasicNotificationsGroup \}

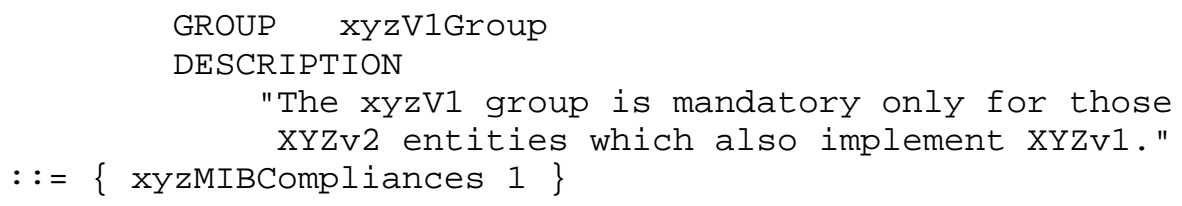


a system must implement the XYZv2-MIB's xyzSystemGroup, xyzStatsGroup, xyzTrapGroup, and xyzSetGroup object conformance groups, as well as the xyzBasicNotificationsGroup notifications group. Furthermore, if the XYZv2 entity also implements XYZv1, then it must also support the XYZvlGroup group, if compliance is to be claimed. 
6. Mapping of the AGENT-CAPABILITIES macro

The AGENT-CAPABILITIES macro is used to convey a set of capabilities present in an agent. It should be noted that the expansion of the AGENT-CAPABILITIES macro is something which conceptually happens during implementation and not during run-time.

When a MIB module is written, it is divided into units of conformance termed groups. If an agent claims to implement a group, then it must implement each and every object, or each and every notification, within that group. Of course, for whatever reason, an agent might implement only a subset of the groups within a MIB module. In addition, the definition of some MIB objects/notifications leave some aspects of the definition to the discretion of an implementor.

Practical experience has demonstrated a need for concisely describing the capabilities of an agent with respect to one or more MIB modules. The AGENT-CAPABILITIES macro allows an agent implementor to describe the precise level of support which an agent claims in regards to a MIB group, and to bind that description to the value of an instance of sysORID [3]. In particular, some objects may have restricted or augmented syntax or access-levels.

If the AGENT-CAPABILITIES invocation is given to a management-station implementor, then that implementor can build management applications which optimize themselves when communicating with a particular agent. For example, the management-station can maintain a database of these invocations. When a management-station interacts with an agent, it retrieves from the agent the values of all instances of sysORID [3]. Based on this, it consults the database to locate each entry matching one of the retrieved values of sysORID. Using the located entries, the management application can now optimize its behavior accordingly.

Note that the AGENT-CAPABILITIES macro specifies refinements or variations with respect to OBJECT-TYPE and NOTIFICATION-TYPE macros in MIB modules, NOT with respect to MODULE-COMPLIANCE macros in compliance statements.

6.1. Mapping of the PRODUCT-RELEASE clause

The PRODUCT-RELEASE clause, which must be present, contains a textual description of the product release which includes this set of capabilities.

6.2. Mapping of the STATUS clause

The STATUS clause, which must be present, indicates whether this 
definition is current or historic.

The value "current" means that the definition is current and valid. The value "obsolete" means the definition is obsolete and this capabilities statement is no longer in use.

6.3. Mapping of the DESCRIPTION clause

The DESCRIPTION clause, which must be present, contains a textual description of this set of capabilities.

\subsection{Mapping of the REFERENCE clause}

The REFERENCE clause, which need not be present, contains a textual cross-reference to some other document, either another information module which defines a related assignment, or some other document which provides additional information relevant to this definition.

6.5. Mapping of the SUPPORTS clause

The SUPPORTS clause, which need not be present, is repeatedly used to name each MIB module for which the agent claims a complete or partial implementation. Each MIB module is named by its module name, and optionally, by its associated OBJECT IDENTIFIER (as registered by the MODULE-IDENTITY macro, see [2]) as well.

6.5.1. Mapping of the INCLUDES clause

The INCLUDES clause, which must follow each and every use of the SUPPORTS clause, is used to name each MIB group associated with the SUPPORTS clause, which the agent claims to implement.

\subsubsection{Mapping of the VARIATION clause}

The VARIATION clause, which need not be present, is repeatedly used to name each object or notification which the agent implements in some variant or refined fashion with respect to the correspondent invocation of the OBJECT-TYPE or NOTIFICATION-TYPE macro.

Note that the variation concept is meant for generic implementation restrictions, e.g., if the variation for an object depends on the values of other objects, then this should be noted in the appropriate DESCRIPTION clause.

By definition, each object specified in a VARIATION clause follows a SUPPORTS clause which names the information module in which that object is defined. Therefore, the use of an IMPORTS statement, to specify from where such objects are imported, is redundant and is not 
required in an information module.

6.5.2.1. Mapping of the SYNTAX clause

The SYNTAX clause, which need not be present, is used to provide a refined SYNTAX for the object named in the correspondent VARIATION clause. Note that if this clause and a WRITE-SYNTAX clause are both present, then this clause only applies when instances of the object named in the correspondent VARIATION clause are read.

Consult Section 9 of [2] for more information on refined syntax.

Note that for enumerated INTEGERs and for the BITS construct, the changes allowed when updating a MIB module include the addition of enumerations and/or changing the labels of existing enumerations (see Section 10.2 of [2]). This type of change can cause problems for an AGENT-CAPABILITIES macro written against the old revision of a MIB module. One way to avoid such problems is to explicitly list all objects having an enumerated syntax in a VARIATION clause, even when all enumerations are currently supported.

\subsubsection{Mapping of the WRITE-SYNTAX clause}

The WRITE-SYNTAX clause, which need not be present, is used to provide a refined SYNTAX for the object named in the correspondent VARIATION clause when instances of that object are written.

Consult section 9 of [2] for more information on refined syntax.

\subsubsection{Mapping of the ACCESS clause}

The ACCESS clause, which need not be present, is used to indicate the agent provides less than the maximal level of access to the object or notification named in the correspondent VARIATION clause.

The only value applicable to notifications is "not-implemented".

The value "not-implemented" indicates the agent does not implement the object or notification, and in the ordering of possible values is equivalent to "not-accessible".

The value "write-only" is provided solely for backward compatibility, and shall not be used for newly-defined object types. In the ordering of possible values, "write-only" is less than "notaccessible". 


\subsubsection{Mapping of the CREATION-REQUIRES clause}

The CREATION-REQUIRES clause, which need not be present, is used to name the columnar objects of a conceptual row to which values must be explicitly assigned, by a management protocol set operation, before the agent will allow the instance of the status column of that row to be set to 'active'. (Consult the definition of Rowstatus [5].)

If the conceptual row does not have a status column (i.e., the objects corresponding to the conceptual table were defined using the mechanisms in $[6,7])$, then the CREATION-REQUIRES clause, which need not be present, is used to name the columnar objects of a conceptual row to which values must be explicitly assigned, by a management protocol set operation, before the agent will create new instances of objects in that row.

This clause must not be present unless the object named in the correspondent VARIATION clause is a conceptual row, i.e., has a syntax which resolves to a SEQUENCE containing columnar objects. The objects named in the value of this clause usually will refer to columnar objects in that row. However, objects unrelated to the conceptual row may also be specified.

All objects which are named in the CREATION-REQUIRES clause for a conceptual row, and which are columnar objects of that row, must have an access level of "read-create".

6.5.2.5. Mapping of the DEFVAL clause

The DEFVAL clause, which need not be present, is used to provide a alternate DEFVAL value for the object named in the correspondent VARIATION clause. The semantics of this value are identical to those of the OBJECT-TYPE macro's DEFVAL clause.

\subsubsection{Mapping of the DESCRIPTION clause}

The DESCRIPTION clause, which must be present for each use of the VARIATION clause, contains a textual description of the variant or refined implementation of the object or notification.

\subsection{Mapping of the AGENT-CAPABILITIES value}

The value of an invocation of the AGENT-CAPABILITIES macro is an OBJECT IDENTIFIER, which names the value of sysORID [3] for which this capabilities statement is valid. 


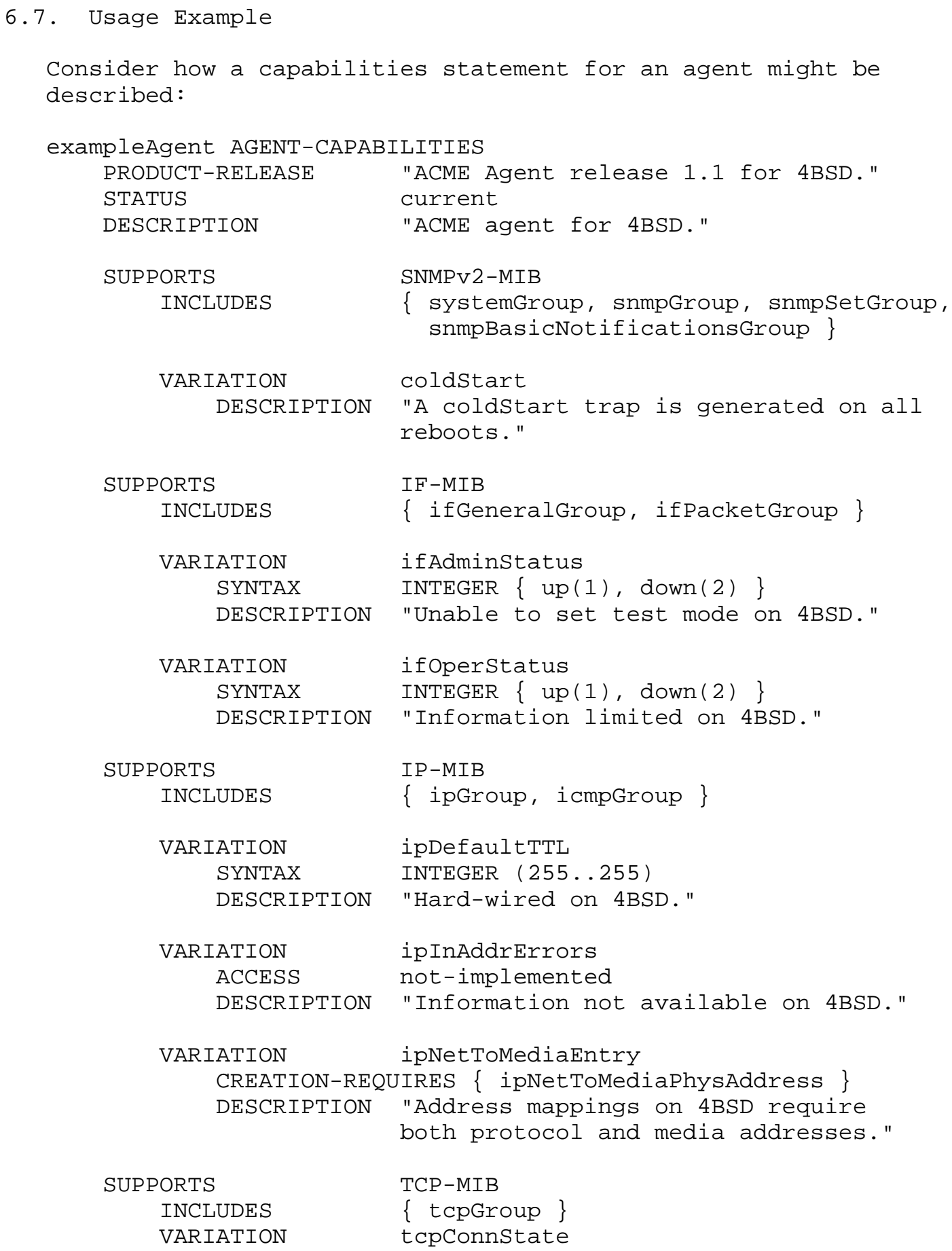




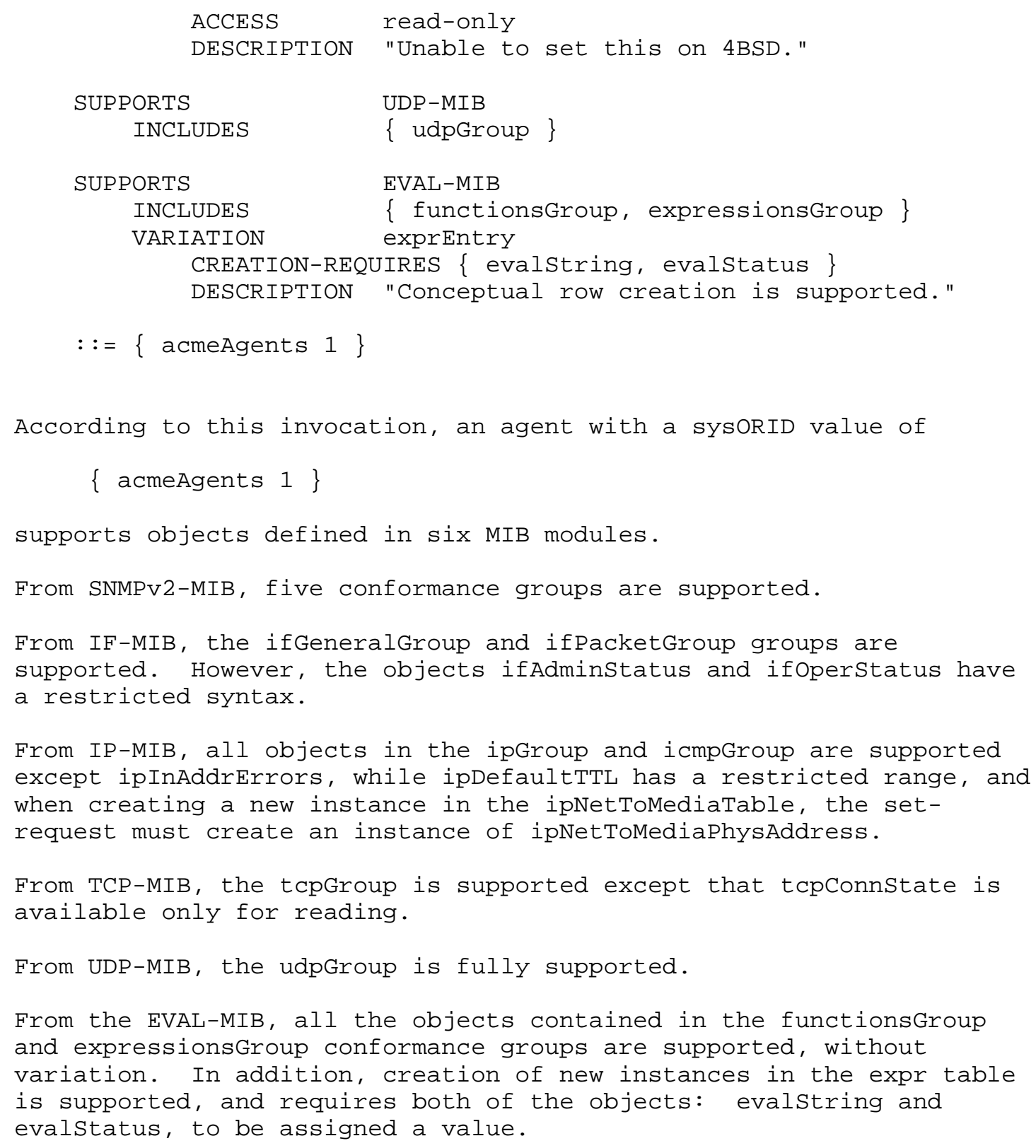


7. Extending an Information Module

As experience is gained with a published information module, it may be desirable to revise that information module.

Section 10 of [2] defines the rules for extending an information module. The remainder of this section defines how conformance groups, compliance statements, and capabilities statements may be extended.

\subsection{Conformance Groups}

It may be desirable to revise the definition of a conformance group (an OBJECT-GROUP or a NOTIFICATION-GROUP) after experience is gained with it. However, conformance groups can be referenced by compliance and/or capabilities definitions. Therefore, a change to a conformance group is not allowed if it has the potential to cause a reference to the group's original definition to be different from a reference to the updated definition. Such changes can only be accommodated by defining a new conformance group with a new descriptor and a new OBJECT IDENTIFIER value.

The following revisions are allowed:

(1) A STATUS clause value of "current" may be revised as "deprecated" or "obsolete". Similarly, a STATUS clause value of "deprecated" may be revised as "obsolete". When making such a change, the DESCRIPTION clause should be updated to explain the rationale.

(2) A REFERENCE clause may be added or updated.

(3) Clarifications and additional information may be included in the DESCRIPTION clause.

(4) Any editorial change.

It is not necessary to change the STATUS value of a conformance group when the status of a member of the group is changed.

\subsection{Compliance Definitions}

It may be desirable to revise the definition of a compliance definition (MODULE-COMPLIANCE) after experience is gained with it. However, changes are not allowed if they cause the requirements specified by the original definition to be different from the requirements of the updated definition. Such changes can only be accommodated by defining a new compliance definition with a new 
descriptor and a new OBJECT IDENTIFIER value.

The following revisions are allowed:

(1) A STATUS clause value of "current" may be revised as "deprecated" or "obsolete". Similarly, a STATUS clause value of "deprecated" may be revised as "obsolete". When making such a change, the DESCRIPTION clause should be updated to explain the rationale.

(2) A REFERENCE clause may be added or updated.

(3) Clarifications and additional information may be included in the DESCRIPTION clause(s).

(4) Any editorial change.

It is not necessary to change the STATUS value of a compliance definition due to a change in the STATUS value of a definition it references.

\subsection{Capabilities Definitions}

It may be desirable to revise the definition of a capabilities definition (AGENT-CAPABILITIES) after experience is gained with it. However, changes are not allowed if they cause the capabilities specified by the original specification to be different from the capabilities of the updated specification. Such changes can only be accommodated by defining a new capabilities definition with a new descriptor and a new OBJECT IDENTIFIER value.

The following revisions are allowed:

(1) A STAtUS clause value of "current" may be revised as "obsolete". When making such a change, the DESCRIPTION clause should be updated to explain the rationale.

(2) A REFERENCE clause may be added or updated.

(3) Clarifications and additional information may be included in the DESCRIPTION clause(s).

(4) Any editorial change.

It is not necessary to change the STATUS value of a capabilities definition due to a change in the STATUS value of a definition it references. 
8. Security Considerations

This document defines the means to define conformance requirements for implementing on documents describing management information. This method of defining conformance requirements has no security impact on the Internet.

9. Editors' Addresses

Keith McCloghrie Cisco Systems, Inc. 170 West Tasman Drive San Jose, CA 95134-1706 USA

Phone: +1 4085265260

EMail: kzmecisco.com

David Perkins

SNMPinfo

3763 Benton street

Santa Clara, CA 95051

USA

Phone: +1408 221-8702

Email: dperkinsasnmpinfo.com

Juergen Schoenwaelder

TU Braunschweig

Bueltenweg 74/75

38106 Braunschweig

Germany

Phone: +49531 391-3283

EMail: schoenwlibr.cs.tu-bs.de 
10. References

[1] Information processing systems - Open systems Interconnection Specification of Abstract Syntax Notation One (ASN.1), International Organization for Standardization. International Standard 8824, (December, 1987).

[2] McCloghrie, K., Perkins, D., Schoenwaelder, J., Case, J., Rose, M. and S. Waldbusser, "Structure of Management Information Version 2 (SMIv2)", STD 58, RFC 2578, April 1999.

[3] The SNMPv2 Working Group, Case, J., McCloghrie, K., Rose, M. and S. Waldbusser, "Management Information Base for Version 2 of the Simple Network Management Protocol (SNMPv2)", RFC 1907, January 1996.

[4] The SNMPv2 Working Group, Case, J., McCloghrie, K., Rose, M. and S. Waldbusser, "Protocol Operations for Version 2 of the Simple Network Management Protocol (SNMPv2)", RFC 1905, January 1996.

[5] McCloghrie, K., Perkins, D., Schoenwaelder, J., Case, J., Rose, M. and S. Waldbusser, "Textual Conventions for SMIv2", STD 58, RFC 2579, April 1999.

[6] Rose, M. and K. McCloghrie, "Structure and Identification of Management Information for TCP/IP-based internets", STD 16, RFC 1155, May 1990 .

[7] Rose, M. and K. McCloghrie, "Concise MIB Definitions", STD 16, RFC 1212, March 1991. 
11. Full Copyright statement

Copyright (C) The Internet Society (1999). All Rights Reserved.

This document and translations of it may be copied and furnished to others, and derivative works that comment on or otherwise explain it or assist in its implementation may be prepared, copied, published and distributed, in whole or in part, without restriction of any kind, provided that the above copyright notice and this paragraph are included on all such copies and derivative works. However, this document itself may not be modified in any way, such as by removing the copyright notice or references to the Internet society or other Internet organizations, except as needed for the purpose of developing Internet standards in which case the procedures for copyrights defined in the Internet standards process must be followed, or as required to translate it into languages other than English.

The limited permissions granted above are perpetual and will not be revoked by the Internet society or its successors or assigns.

This document and the information contained herein is provided on an "AS IS" basis and THE INTERNET SOCIETY AND THE INTERNET ENGINEERING TASK FORCE DISCLAIMS ALL WARRANTIES, EXPRESS OR IMPLIED, INCLUDING BUT NOT LIMITED TO ANY WARRANTY THAT THE USE OF THE INFORMATION HEREIN WILL NOT INFRINGE ANY RIGHTS OR ANY IMPLIED WARRANTIES OF MERCHANTABILITY OR FITNESS FOR A PARTICULAR PURPOSE." 